\title{
Observation of different phases during an ELM crash with the help of nitrogen seeding
}

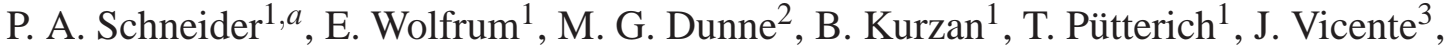 \\ R. Wenninger ${ }^{1}$ and the ASDEX Upgrade Team ${ }^{1}$ \\ ${ }^{1}$ Max-Planck-Institut für Plasmaphysik, EURATOM Association, Garching, Germany \\ ${ }^{2}$ Department of Physics, University College Cork, Association Euratom-DCU, Cork, Ireland \\ ${ }^{3}$ Associação EURATOM/IST, Instituto de Plasmas e Fusão Nuclear, Instituto Superior Técnico I \\ Universidade Técnica de Lisboa, Lisboa, Portugal \\ a e-mail: philip.schneider@ipp.mpg.de
}

Edge localised modes ELMs are of importance for the scenario development in reactor like conditions. Their beneficial influence on the plasma, like impurity removal, needs to be maintained, while negative effects, such as large heat loads on plasma facing components, have to be mitigated. A first principle understanding of the processes involved would open up new possibilities in the optimisation process. However, such an understanding is hampered by the lack of the required diagnostic capabilities. This lack is caused by the extreme nature of an ELM, namely the short timescales of sub ms and the small spatial scales which go down to a few mm. Another reason is the importance of the local current density for which routinely used reconstruction methods were only recently developed [1].

Impurity seeding in plasma discharges, in particular nitrogen seeding, is applied mainly to increase radiative cooling in the divertor region. However, other side effects were observed, notably improved energy confinement [2] and changed ELM behaviour [3].

The influence of nitrogen seeding on ELMs is utilized in this paper to obtain information about the ELM crash itself. For comparison, two consecutive discharges with exactly the same parameters $\left(I_{\mathrm{p}}=1.0 \mathrm{MA}, B_{\mathrm{t}}=2.5 \mathrm{~T}, P_{\mathrm{NBI}}=7.3 \mathrm{MW}, P_{\mathrm{ECRH}}=0.7 \mathrm{MW}\right.$ and $\left.\delta=0.25\right)$ were conducted in the full tungsten machine ASDEX Upgrade. The only difference in the discharge pair was a nitrogen gas puff in the divertor during the second discharge. This nitrogen gas puff was applied in addition to a deuterium gas puff of the same magnitude. Several time traces of important quantities are compared for both discharges in Fig. 1. (a) and (c) show that the discharge settings where the same; (d) shows that no change in the central line averaged density was observed while the density at the edge is reduced by $\sim 10 \%$; (b) the plasma stored energy is initially similar in both cases. When the deuterium gas puff was reduced and higher heating power was applied, confinement in the nitrogen seeded case is increased; (e) shows the envelope of a Mirnov coil measurement and illustrates that also the core MHD behaviour is similar in both cases. The additional nitrogen in the plasma influences the effective charge number $Z_{\text {eff. }}$ This influence was studied in detail in [4] and showed that until $3.0 \mathrm{~s}$ the additional nitrogen does not increase $Z_{\text {eff }}$, only with the onset of the 3 rd NI beam source $Z_{\text {eff }}$ rises by $40 \%$ at the plasma edge for the nitrogen seeded case. In the plasma center $Z_{\text {eff }}$ is the same for both cases throughout the discharge.

By comparing the discharges a remarkable change in the edge dynamics is also observed. In the case with nitrogen puff the ELMs appear to be shorter, have a faster repetition rate and have less impact on the plasma edge than the ELMs in the reference case. In general an ELM is characterized only by a collapse of the pedestal $(\sim 1 \mathrm{~ms})$ and a recovery phase $(\geq 5 \mathrm{~ms})$. In Fig. 2 it becomes clear that more phases during the ELM cycle are necessary to describe the differences between "nitrogen ELMs" and regular type-I ELMs. All traces in Fig. 2 show an 


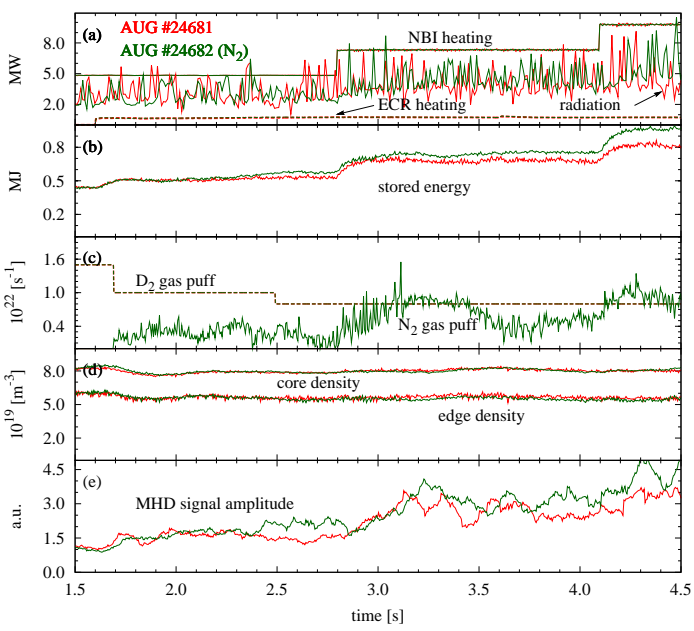

Figure 1: Time traces for two comparison discharges one without nitrogen seeding (red) and one with nitrogen seeding (green).

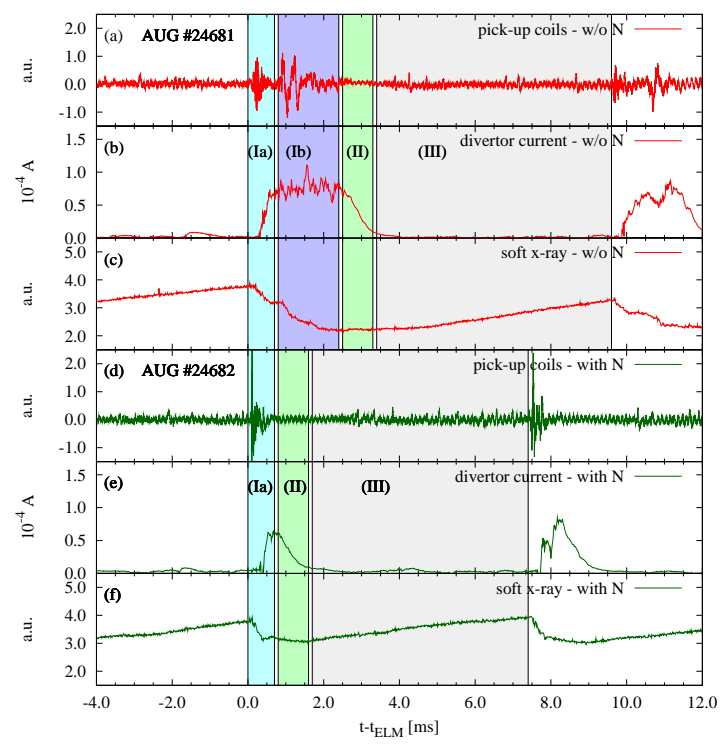

Figure 2: The time traces relative to the onset of an ELM. Different phases in the ELM cycle are highlighted.

example ELM around $2.4 \mathrm{~s}$ in the discharges. Fig. 2 (a)-(c) show the time traces of magnetic perturbation pick-up coils, the divertor thermo-currents and edge soft x-ray measurements for the reference case without nitrogen gas puff. The thermo-current is induced by the temperature difference between the inner and outer divertor targets. (d)-(f) show the same diagnostics in the case with nitrogen gas puff. With these traces three different phases are identified in the ELM cycle.

(I) the ELM crash: energy and particles are lost from the plasma edge. This is best visible in the soft x-ray edge channel (c),(f) where the radiation drops during the whole phase (1). During the same time broad band magnetic oscillations from $10-400 \mathrm{kHz}$ are measured by the magnetic pick-up coils (a),(d). The lost particles are measured a few hundred $\mu \mathrm{s}$ later in the divertor (b), (e).

(II) intermediate phase: the strong magnetic perturbation of the plasma edge has stopped and is replaced by a quiescent phase (a),(d) and the soft x-ray radiation stays constant (c),(f). Thermo-currents exhibit a decreasing signal as the divertor plasma cools down on a $1 \mathrm{~ms}$ timescale after the impact of the ELM (b),(e).

(III) recovery phase: the pedestal recovers and the soft $x$-ray radiation (c),(f) increases until the next ELM crash. The pick-up coils indicate perturbations above the level of the intermediate phase but well below the level during the ELM crash. The divertor currents show only a background signal, indicative of a minor temperature difference between the divertor targets.

In this classification both cases behave identically. Differences arise during the crash phase where the type-I ELM can be divided into two distinct events (Ia) and (Ib) while the nitrogen ELM shows only one (Ia). The first crash phase (Ia) exhibits the same characteristics on identical 

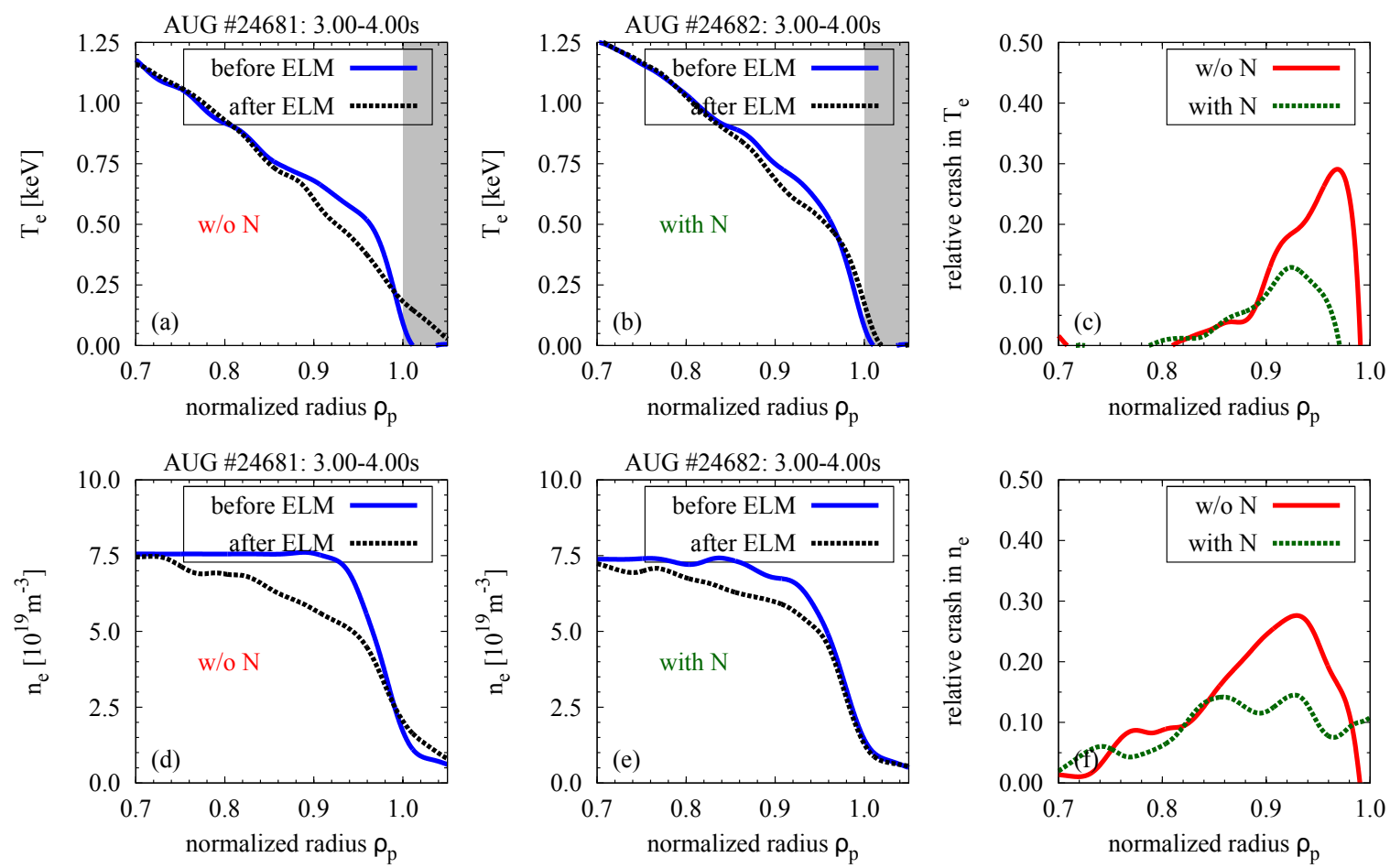

Figure 3: ELM synchronised profiles of electron temperature (a)-(d) and electron density (e)-(f) before (blue,solid) and after (black,dashed) an ELM.

time scales in both cases. It starts with a fast $(\sim 0.5 \mathrm{~ms})$ drop in the soft $\mathrm{x}$-ray radiation $(\mathrm{c}),(\mathrm{f})$ followed by a short (0.3-0.4 ms) saturation phase. In the same time a broad band oscillation is visible in the magnetic signal (a),(d). This oscillation is only visible in the phase (Ia). For the nitrogen ELM phase (Ia) is directly followed by the intermediate phase (II). In the case of typeI ELMs a second crash phase (Ib) can be identified. During this phase the soft x-ray radiation is further reduced (c). The magnetic signal still shows broad band oscillations, however, with reduced amplitude and they are overlaid with a strong oscillation of $<10 \mathrm{kHz}$ which appears as several spikes (a). During the phase (Ib) there is a steady flow of particles into the divertor which lasts 1-2 ms, as evidenced by the divertor current (b).

Profiles of temperature and density prior and after the crash suggest that the region affected by the ELM in the presence nitrogen seeding is located on top of the pedestal and the steep gradient region is not influenced. The type-I ELM affects the profiles on a broader region which includes the very edge with steep gradients and the SOL. This is documented in detail in Fig. 3. The profiles for temperature (a),(b) and density (d),(e) are shown directly before the ELM crash (blue, solid) and immediately after the crash (black, dashed). The profiles are obtained with ELM synchronised averaging over $1 \mathrm{~s}$ which means 100-200 ELMs. The intervals used for synchronisation are $0.5 \mathrm{~ms}$ long and taken relative to an ELM.

In the case with nitrogen (Fig. 3 (b)) the ELM triggers only a small drop in the electron temperature from $0.80<\rho_{\mathrm{p}}<0.96$ while the steep gradient is preserved until after the ELM. The reference case Fig. 3 (a) shows the collapse of the whole transport barrier. This is also observed for the electron density in Fig. 3 (d),(e). While the transport barrier collapses in the reference case (d) it remains unaffected for the case with nitrogen (e). Furthermore, the density increases in the SOL for the reference case while it stays the same with nitrogen. The difference between density and temperature is that the crash in $n_{\mathrm{e}}$ extends further into the plasma - up to $\rho_{\mathrm{p}} \sim 0.75$. 
The relative change of $T_{\mathrm{e}}$ (c) and $n_{\mathrm{e}}$ (f) caused by the crash gives an indication of this ELM affected area. In both cases the profiles inside $\rho_{\mathrm{p}}=0.85-0.90$ are affected to the same extent by the crash. This is observed for both temperature and density. The only differences are visible outside this region and are most pronounced in the steep gradient region. With addition of nitrogen a maximal drop of $\sim 15 \%$ is observed in $T_{\mathrm{e}}$ and $n_{\mathrm{e}}$, while a $\sim 30 \%$ drop in the reference case is observed.

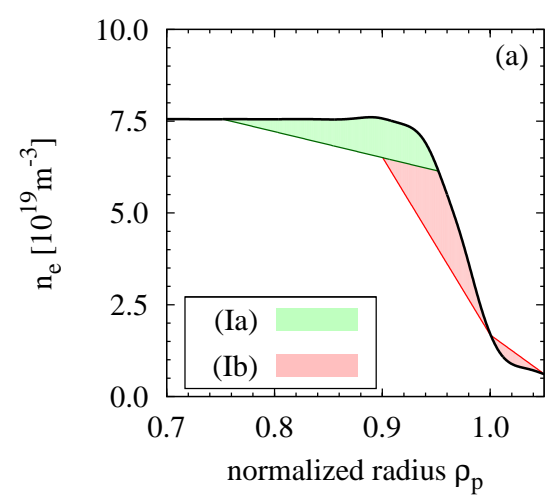

Figure 4: A sketch of the possible affected area due to different crash phases.

Combining both observations, the averaged profile measurements with high spatial resolution and the highly temporally resolved time traces of magnetic pick-up coils and soft x-ray diodes, one obtains a picture of the ELM crash which would not be possible with the individual measurements. The profiles from the nitrogen seeded discharge correspond to the crash phase (Ia) in Fig. 2. Assuming that the phase (Ia) of the seeded case and the reference case are the same, as their time traces suggest, the profile response of crash phase (Ib) can be deduced from the profiles, in the reference case. The result is illustrated in Fig. 4 as a sketch. In a first crash particles and energy are only expelled from a region spanning from the pedestal top inwards. In a separated second crash the transport barrier also collapses and more particles and energy are lost from the plasma edge. The absolute energy loss during both phases is comparable.

The observations in the different time traces suggest two different mechanisms for the loss of energy and particles in the pedestal. Considering the observations of strongly reduced filament activity in the presence of nitrogen [3] it is likely that the second crash (Ib) is related to filamentary losses in the pedestal. This could also explain the bursty nature of the magnetics signal. The type-I ELMs known from AUG still with Carbon wall have more characteristics in common with the type-I ELMs considered here than with the nitrogen ELMs. The addition of nitrogen to the plasma stabilised the edge pedestal against the (Ib) instability. This consequently leads to an increase of the ELM frequency because the time for the second crash does not occur and the recovery can be faster. The key quantity for the stabilisation of (Ib) could not be identified in the available discharge set. A direct influence of the effective charge number seems unlikely, since different ELM characteristics are observed before differences in $Z_{\mathrm{eff}}$ are measured. The largest differences were observed for quantities dependent on the ratio of $n_{\mathrm{e}} / T_{\mathrm{e}}$ while quantities with the product $n_{\mathrm{e}} T_{\mathrm{e}}$ varied less. Candidates are e.g. the collisionality or the plasma resistivity. The effect of the nitrogen seeding on the collisionality depends on the change in $Z_{\text {eff }}$, while the resistivity is decreased in all cases.

The "ELM"-crash can be constituted out of multiple phases and these phases exhibit fundamental differences. To understand the processes involved, it will be important to treat the different phenomena separately.

\section{References}

[1] DUNNE, M. G. et al., Nuclear Fusion 52 (2012) 123014.

[2] SCHWEINZER, J. et al., Nuclear Fusion 51 (2011) 113003.

[3] MüLLER, H. et al., Nuclear Fusion 51 (2011) 073023.

[4] RATHGEBER, S. K. et al., Plasma Physics and Controlled Fusion 52 (2010) 095008. 